Eur. J. Math. Appl. (2021)1:15

URL: http://ejma.euap.org

(C) 2021 European Journal of Mathematics and Applications

\title{
QUALITATIVE BEHAVIOR OF A SYSTEM OF SECOND ORDER DIFFERENCE EQUATIONS
}

\author{
E. M. ELSAYED ${ }^{1,2}$ AND A. ALSHAREEF ${ }^{1, *}$
}

ABSTRACT. In this paper, we deal with the following system of rational difference equations

$$
\chi_{n+1}=\frac{\alpha_{1} \chi_{n-1} \tau_{n-1}}{\beta+\tau_{n-1}} \text { and } \tau_{n+1}=\frac{\alpha_{2} \chi_{n} \tau_{n}}{\beta+\chi_{n}},
$$

where $\alpha_{1}, \alpha_{2}$ and $\beta$ are real positive numbers and the initial conditions are $\chi_{0}, \chi_{-1}, \tau_{0}$ and $\tau_{-1}$. We show that the solutions of this system are bounded. Also, we prove that there is no periodic solutions of period two. Moreover, we investigate the local and global stability of the equilibrium point. Some numerical examples are given.

\section{INTRODUCTION}

In recent years, a lot of articles focus on investigating the system of rational difference equations, since it appears in many fields of sciences and it analogy of a discrete system. The study of a system of rational difference equation concern to explore the solution behavior by attempting to find the critical points and study their stability, analyze the boundedness, examine the periodic solution and sometimes present the explicit solution if it is possible. Kurbanli [15] et al. investigated the solutions of the below system of fractional difference equations

$$
W_{n+1}=\frac{W_{n-1}}{Z_{n} W_{n-1}+1} \quad \text { and } \quad Z_{n+1}=\frac{Z_{n-1}}{W_{n} Z_{n-1}+1} .
$$

In [2], Al-Hadibat et al. solved and discussed the bifurcation analysis for the following system

$$
W_{n+1}=\frac{W_{n-1}}{W_{n-1}+r} \quad \text { and } \quad Z_{n+1}=\frac{W_{n-1} Z_{n}}{W_{n-1} Z_{n}+r} .
$$

Haddad et al. [10] presented the explicit solution of the rational difference equations

$$
W_{n+1}=\frac{r W_{n} Z_{n-1}}{Z_{n}-a}+b \quad \text { and } \quad Z_{n+1}=\frac{t W_{n-1} Z_{n}}{W_{n}-b}+a .
$$

Kara and Yazilk [11] proved that the following rational difference of higher order can be solve

$$
W_{n}=\frac{W_{n-k} Z_{n-k-l}}{Z_{n-l}\left(\alpha_{n}+\beta_{n} W_{n-k} Z_{n-k-l}\right)} \quad \text { and } \quad Z_{n}=\frac{Z_{n-k} W_{n-k-l}}{W_{n-l}\left(A_{n}+B_{n} Z_{n-k} W_{n-k-l}\right)}
$$

\footnotetext{
${ }^{1}$ Mathematics Department, Faculty of Science, King Abdulaziz University, , P. O. Box 80203, JEDDAH 21589, SAUdi ARABiA

${ }^{2}$ Department of Mathematics, Faculty of Science,, Mansoura University, Mansoura 35516 , EGYPT

*Corresponding AUthor

E-mail addresses: emmelsayed@yahoo.com, 3beer23sh@gmail.com.

Key words and phrases. difference equation; periodicity; critical points; stability; global attractor.

Received 03/10/2021.
} 
In [5], Elsayed obtained the solution of the below rational difference system of order two

$$
W_{n+1}=\frac{W_{n-1}}{ \pm 1+W_{n-1} Z_{n}} \quad \text { and } \quad Z_{n+1}=\frac{Z_{n-1}}{\mp 1+Z_{n-1} W_{n}} .
$$

To more studied in nonlinear difference equation, we refer to see $[1,3,6,7,9,12-14,16]$. The main purpose of this article is to study the character of the solution behavior for the following system of second order

$$
\chi_{n+1}=\frac{\alpha_{1} \chi_{n-1} \tau_{n-1}}{\beta+\tau_{n-1}} \text { and } \tau_{n+1}=\frac{\alpha_{2} \chi_{n} \tau_{n}}{\beta+\chi_{n}},
$$

where $\alpha_{1}, \alpha_{2}$ and $\beta$ are positive real numbers and the initial conditions are $\chi_{0}, \chi_{-1}, \tau_{0}$ and $\tau_{-1}$. This article was collected as follows: In Section 2, we introduce some definitions and theorems that are used for our study. In Section 3, we analyze the boundedness of the solution. After that, we examine the nature of the periodic solution in Section 4. In Section 5 and Section 6 , we study the local and global stability of the equilibrium points, respectively. Finally, in Section 7, we present some numerical examples in order to illustrate the theoretical results.

\section{PReliminaries AND Definitions}

This section deals with theorems and definitions that are useful in our study.

Definition 2.1. Let $V \in \mathbb{R}$ and $H$ is a continuous function and it has partial derivative on $V^{k+1}$, such that $V^{k+1}=V \times V \times \ldots \times V(k+1$-times $)$. Then, for $v_{-l}, v_{-k+1}, \ldots, v_{0} \in V$, the difference equation

$$
v_{n+1}=H\left(v_{n}, v_{n-1}, \ldots, v_{n-k}\right), \quad n=0,1, \ldots
$$

has a unique solution $\left\{v_{n}\right\}_{n=-k}^{\infty}$.

Definition 2.2. A point $\bar{v}$ is said to be an equilibrium point of (2) if $\bar{v}=H(\bar{v})$ is satisfied.

Definition 2.3. The equilibrium point $\bar{v}$ of (2) is stable when

$$
\forall \xi>0, \exists \eta>0 \text {, s.t., }\left|v_{0}-\bar{v}\right|<\eta \text {, implies that }\left|H^{n}\left(v_{0}\right)-\bar{v}\right|<\xi,
$$

$\forall n>0$. And it is unstable if $\bar{v}$ is not stable.

Definition 2.4. The point $\bar{v}$ is said to be attracting when the relation

$$
\exists \vartheta>0 \text {, s.t., }\left|v_{0}-\bar{v}\right|<\vartheta \text {, implies that } \lim _{n \longrightarrow \infty} v_{n}=\bar{v} .
$$

is true. If $\vartheta=\infty$, then $\bar{v}$ is global attractor.

Definition 2.5. The following statements are holds for (2):

(i) If $\bar{v}$ is stable and attracting, then it is a asymptotically stable equilibrium point.

(ii) $\bar{v}$ is said to be a globally asymptotically stable equilibrium point when it is stable and globally attracting.

Definition 2.6. Consider a system of difference equation is defined as

$$
v(n+1)=H(v(n)), \quad v \in U \text { and } H(0)=0,
$$

since $U$ is a neighbourhood of the origin in $\mathbb{R}^{n}$. 
Theorem 2.1. Suppose that $F$-continuous differentiable on open neighbourhood, $\mathbf{G} \subseteq \mathbb{R}^{k+1}$. Then

(i) If all the characteristic roots of (3) lie inside the unit disk, then $\bar{v}$ is asymptotically stable.

(ii) If at least one characteristic roots of (3) outside the unit disk, then $\bar{v}$ is unstable.

Theorem 2.2. [8] Assume that the polynomial equation is

$$
x_{4} Y^{4}+x_{3} Y^{3}+x_{2} Y^{2}+x_{1} Y+x_{0}=0 .
$$

Such that $x_{i} \in \mathbb{R}$ for $i=0,1, \ldots, 4$. Then, the sufficient and necessary conditions that all eigenvalues of (4) are less that one, i. e., all roots of (4) are inside the unit disk, are

(i) $\left|x_{1}+x_{3}\right|<1+x_{0}+x_{2}$.

(ii) $\left|x_{1}-x_{3}\right|<2\left(1-x_{0}\right)$.

(iii) $x_{2}-3 x_{0}<3$.

(iv) $x_{0}+x_{2}+x_{0}^{2}+x_{1}^{2}+x_{0}^{2} x_{2}+x_{0} x_{3}^{2}<1+2 x_{0} x_{2}+x_{1} x_{3}+x_{0} x_{1} x_{3}+x_{0}^{3}$.

Theorem 2.3. [4] Suppose that $W:[a, b]^{h+1} \longrightarrow[a, b]$ is a continuous function, where $h>0$ and $[a, b] \in \mathbb{R}$. Let the difference equation be

$$
u_{n+1}=W\left(u_{n}, u_{n-1}, \ldots, u_{n-h}\right), \quad n=0,1, \ldots
$$

Consider $W$ is weakly monotonic. Suppose, in addition that, wherever $(l, L)$ is a solution of the system

$$
l=W\left(l_{1}, l_{2}, \ldots, l_{k+1}\right) \text { and } L=W\left(L_{1}, L_{2}, \ldots, L_{k+1}\right),
$$

where

$$
\begin{aligned}
& l_{i}= \begin{cases}l, & \text { if } \quad W \text { nondecreasing in } \mathbb{Z}_{i}, \\
L, & \text { if } \quad W \text { nonincreasing in } \mathbb{Z}_{i},\end{cases} \\
& L_{i}= \begin{cases}L, & \text { if } \quad W \text { nondecreasing in } \mathbb{Z}_{i}, \\
l, & \text { if } \quad W \text { nonincreasing in } \mathbb{Z}_{i} .\end{cases}
\end{aligned}
$$

Thus, $l=L . \forall i=1,2, \ldots, l+1$. Then there exists a unique equilibrium point and it is globally attractor for equation (5).

Definition 2.7. The sequence $\left\{u_{n}\right\}_{n=-j}^{\infty}$ has a periodic solution with period $t$ if $u_{n+t}=$ $u_{n} \forall n \geq-j$.

\section{Boundedness of the SOlution}

The next theorem analysis the boundedness of every non-negative solutions of system (1).

Theorem 3.1. The solutions $\left\{\left(\chi_{n}, \tau_{n}\right)\right\}_{n=-1}^{\infty}$ of system (1) are bounded if and only if $\alpha_{1}$ and $\alpha_{2}$ are less than one.

Proof. For any non-negative solution $\left\{\left(\chi_{n}, \tau_{n}\right)\right\}_{n=-1}^{\infty}$ of (1), one has

$$
\chi_{n+1}=\frac{\alpha_{1} \chi_{n-1} \tau_{n-1}}{\beta+\tau_{n-1}} \leq \frac{\alpha_{1} \chi_{n-1} \tau_{n-1}}{\tau_{n-1}} \Rightarrow \chi_{n+1} \leq \alpha_{1} \chi_{n-1},
$$


Then, for $n=0,1, \ldots .$. , we see that

$$
\begin{aligned}
n= & 0 \Rightarrow \chi_{1} \leq \alpha_{1} \chi_{-1}, \\
n= & \Rightarrow \chi_{2} \leq \alpha_{1} \chi_{0}, \\
n= & \Rightarrow \chi_{3} \leq \alpha_{1} \chi_{1} \leq\left(\alpha_{1}\right)^{2} \chi_{-1}, \\
n= & \Rightarrow \chi_{4} \leq \alpha_{1} \chi_{2} \leq\left(\alpha_{1}\right)^{2} \chi_{0}, \\
n= & \Rightarrow \chi_{5} \leq \alpha_{1} \chi_{3} \leq\left(\alpha_{1}\right)^{3} \chi_{-1}, \\
n= & 5 \Rightarrow \chi_{6} \leq \alpha_{1} \chi_{4} \leq\left(\alpha_{1}\right)^{3} \chi_{0}, \\
\vdots & \vdots \\
n= & 2 N-1 \Rightarrow \chi_{n} \leq\left(\alpha_{1}\right)^{N} \chi_{-1}, \quad N=0,1,2, \ldots \\
n= & \Rightarrow \chi_{n} \leq\left(\alpha_{1}\right)^{N} \chi_{0}, \quad N=0,1,2, \ldots
\end{aligned}
$$

Therefore, the sequence $\left\{\chi_{n}\right\}_{n=-1}^{\infty}$ is bounded above by $\rho_{1}$ which is given as $\rho_{1}=$ $\max \left\{\alpha_{1}^{N} \chi_{0}, \alpha_{1}^{N} \chi_{-1}\right\}$ for $N=0,1,2, .$. when $\alpha_{1}<1$. Similarly, we show that the sequence $\left\{\tau_{n}\right\}_{n=-1}^{\infty}$ is bounded by using the following relation

$$
\tau_{n+1}=\frac{\alpha_{2} \chi_{n} \tau_{n}}{\beta+\chi_{n}} \leq \frac{\alpha_{2} \chi_{n} \tau_{n}}{\chi_{n}} \Rightarrow \tau_{n+1} \leq \alpha_{2} \tau_{n}
$$

Then, for $n=-1,0,1, \ldots$, we get

$$
\begin{aligned}
n= & -1 \Rightarrow \tau_{0} \leq \alpha_{2} \tau_{-1}, \\
n= & 0 \Rightarrow \tau_{1} \leq \alpha_{2}^{2} \tau_{-1}, \\
n= & 1 \Rightarrow \tau_{2} \leq \alpha_{2} \tau_{1} \leq\left(\alpha_{2}\right)^{3} \tau_{-1}, \\
n= & 2 \Rightarrow \tau_{3} \leq \alpha_{2} \tau_{2} \leq\left(\alpha_{2}\right)^{4} \tau_{-1}, \\
\vdots & \vdots \\
n= & N \Rightarrow \tau_{n} \leq\left(\alpha_{2}\right)^{N+1} \tau_{-1}, \quad N=0,1,2, \ldots
\end{aligned}
$$

It is clear that if $\alpha_{2}<1$, then the sequence $\left\{\tau_{n}\right\}_{n=-1}^{\infty}$ is bounded from above by $\sigma_{1}=$ $\left(\alpha_{2}\right)^{N+1} \tau_{-1}$ for $N=0,1,2, \ldots$ Furthermore, we will prove that the solutions $\left\{\left(\chi_{n}, \tau_{n}\right)\right\}_{n=-1}^{\infty}$ are bounded. Thus, from (1) we have

$$
\chi_{n+1} \geq \frac{\alpha_{1}}{\beta+\tau_{n-1}} \Rightarrow \chi_{n} \geq \frac{\alpha_{1}}{\beta+\sigma_{1}}, \quad \forall n=-1,0,1, \ldots
$$

Implies $\rho_{2} \leq \chi_{n} \leq \rho_{1}$, where $\rho_{2}=\frac{\alpha_{1}}{\beta+\sigma_{1}}$. Moreover, we get the equation

$$
\tau_{n+1} \geq \frac{\alpha_{2}}{\beta+\chi_{n}} \Rightarrow \tau_{n} \geq \frac{\alpha_{2}}{\beta+\rho_{1}}, \quad \forall n=-1,0,1, \ldots
$$

Hence, we get $\sigma_{2} \leq \tau_{n} \leq \sigma_{1}$, since $\sigma_{2}=\frac{\alpha_{2}}{\beta+\rho_{1}}$.

\section{Periodic SOlution}

In this section, we discuss the periodic solution of period two.

Theorem 4.1. There is no periodic solution of period two for the system (1). 
Proof. Assume there exist a priodic solution of period two of (1)

$$
\ldots,\left(\Theta_{1}, \Lambda_{1}\right),\left(\Theta_{2}, \Lambda_{2}\right),\left(\Theta_{1}, \Lambda_{1}\right),\left(\Theta_{2}, \Lambda_{2}\right), \ldots
$$

Then, from (1), we have

$$
\begin{aligned}
\Theta_{1}=\frac{\alpha_{1} \Theta_{1} \Lambda_{1}}{\beta+\Lambda_{1}} \quad \Rightarrow \beta \Theta_{1}+\Theta_{1} \Lambda_{1}=\alpha_{1} \Theta_{1} \Lambda_{1}, \\
\Theta_{2}=\frac{\alpha_{1} \Theta_{2} \Lambda_{2}}{\beta+\Lambda_{2}} \quad \Rightarrow \beta \Theta_{2}+\Theta_{2} \Lambda_{2}=\alpha_{1} \Theta_{2} \Lambda_{2}, \\
\Lambda_{1}=\frac{\alpha_{2} \Theta_{2} \Lambda_{2}}{\beta+\Theta_{2}} \quad \Rightarrow \beta \Lambda_{1}+\Lambda_{1} \Theta_{2}=\alpha_{2} \Theta_{2} \Lambda_{2}, \\
\Lambda_{2}=\frac{\alpha_{2} \Theta_{1} \Lambda_{1}}{\beta+\Theta_{1}} \Rightarrow \beta \Lambda_{2}+\Lambda_{2} \Theta_{1}=\alpha_{2} \Theta_{1} \Lambda_{1} .
\end{aligned}
$$

Multiplying equations (6) and (7) by $\Theta_{2} \Lambda_{2}$ and $\Theta_{1} \Lambda_{1}$, respectively.

$$
\begin{aligned}
& \beta \Theta_{2} \Lambda_{2} \Theta_{1}+\left(1-\alpha_{1}\right) \Theta_{2} \Lambda_{2} \Theta_{1} \Lambda_{1}=0, \\
& \beta \Theta_{1} \Lambda_{1} \Theta_{2}+\left(1-\alpha_{1}\right) \Theta_{1} \Lambda_{1} \Theta_{2} \Lambda_{2}=0 .
\end{aligned}
$$

By subtracting (11) from (10), we get

$$
\begin{aligned}
\beta\left(\Theta_{2} \Lambda_{2} \Theta_{1}-\Theta_{1} \Lambda_{1} \Theta_{2}\right) & =0 \\
\Theta_{2} \Lambda_{2} \Theta_{1} & =\Theta_{1} \Lambda_{1} \Theta_{2} .
\end{aligned}
$$

Hence, we see that $\Lambda_{1}=\Lambda_{2}$. Now, to show that $\Theta_{1}=\Theta_{2}$, we multiply (8) by $\Theta_{1} \Lambda_{2}$ and (9) by $\Theta_{2} \Lambda_{1}$,

$$
\begin{aligned}
& \beta \Theta_{1} \Lambda_{2} \Lambda_{1}+\Theta_{1} \Lambda_{2} \Lambda_{1} \Theta_{2}=\alpha_{2} \Theta_{2} \Theta_{1} \Lambda_{2}^{2} \text {, } \\
& \beta \Theta_{2} \Lambda_{1} \Lambda_{2}+\Theta_{2} \Lambda_{1} \Lambda_{2} \Theta_{1}=\alpha_{2} \Theta_{2} \Theta_{1} \Lambda_{1}^{2} \text {. }
\end{aligned}
$$

Then, from (12) and (13), we have

$$
\beta \Lambda_{1} \Lambda_{2}\left(\Theta_{1}-\Theta_{2}\right)+\alpha_{2} \Theta_{1} \Theta_{2}\left(\Lambda_{1}^{2}-\Lambda_{2}^{2}\right)=0 .
$$

Since $\Lambda_{1}=\Lambda_{2}$, the equation (14) gives $\Theta_{1}=\Theta_{2}$. Therefore, the periodic solution of period two is not exist.

\section{LOCAL STABILITY OF EQUILIBRIA}

By solving algebraic equations

$$
\bar{\chi}=\frac{\alpha_{1} \bar{\chi} \bar{\tau}}{\beta+\bar{\tau}}=0 \quad \text { and } \quad \bar{\tau}=\frac{\alpha_{2} \bar{\chi} \bar{\tau}}{\beta+\bar{\chi}}=0,
$$

we have

$$
E_{0}=(\bar{\chi}, \bar{\tau})=(0,0) \quad \text { and } \quad E_{1}=(\bar{\chi}, \bar{\tau})=\left(\frac{\beta}{\alpha_{2}-1}, \frac{\beta}{\alpha_{1}-1}\right) .
$$

We notice that positive equilibrium point $E_{1}$ exists if and only if $\alpha_{i}>1$, where $i=1,2$. In the next theorem, we explore the stability analysis of the equilibrium points.

Theorem 5.1. For system (1), the following statements are holds.

(a) The equilibrium point $E_{0}$ is always exists and it is locally asymptotic stable.

(b) If $\alpha_{i}>1$, then the positive fixed point $E_{1}$ exists and it is unstable. 
Proof. To investigate the stability of the critical points, we assume $\chi_{n-1}=\omega_{n}$ and $\tau_{n-1}=v_{n}$, then the system (1) will be

$$
\left(\chi_{n+1}, \omega_{n+1}, \tau_{n+1}, v_{n+1}\right)^{T}=\left(\frac{\alpha_{1} \omega_{n} v_{n}}{\beta+v_{n}}, \chi_{n}, \frac{\alpha_{2} \chi_{n} \tau_{n}}{\beta+\chi_{n}}, \tau_{n}\right)^{T} .
$$

The Jacobian matrix of $(16)$ is $J F=\left(\begin{array}{cccc}0 & \frac{\alpha_{1} v_{n}}{\beta+v_{n}} & 0 & \frac{\alpha_{1} \beta \omega_{n}}{\left(\beta+v_{n}\right)^{2}} \\ 1 & 0 & 0 & 0 \\ \frac{\alpha_{2} \beta \tau_{n}}{\left(\beta+\chi_{n}\right)^{2}} & 0 & \frac{\alpha_{2} \chi_{n}}{\beta+\chi_{n}} & 0 \\ 0 & 0 & 1 & 0\end{array}\right)$. By evaluating $J F$ at $E_{0}=(0,0,0,0)$, we get the eigenvalues $\left|\lambda_{1}\right|=\left|\lambda_{2}\right|=\left|\lambda_{3}\right|=\left|\lambda_{4}\right|=0$. Clearly, all the eigenvalues are inside the unit disk. It means that the origin is locally stable. The proof of (a) is complete. Now, we will apply Jury's condition to prove (b). The characteristic equation $\Phi(\Lambda)$ of Jacobian matrix $\left.J F\right|_{E_{1}}$, which is evaluated at $E_{1}$, defined as

$$
\Phi(\Lambda)=\Lambda^{4}-\Lambda^{3}-\Lambda^{2}+\frac{\alpha_{1}+\alpha_{2}-1}{\alpha_{1} \alpha_{2}} \Lambda
$$

Such that $z_{4}=1, z_{3}=-1, z_{2}=-1, z_{1}=\frac{\alpha_{1}+\alpha_{2}-1}{\alpha_{1} \alpha_{2}}$ and $z_{0}=0$. Now, we examine condition (i) of Theorem. 2.2

$$
\begin{aligned}
\left|z_{1}+z_{3}\right| & <1+z_{0}+z_{2}, \\
\left|\frac{\alpha_{1}+\alpha_{2}-1}{\alpha_{1} \alpha_{2}}-1\right| & \nless 0, \\
\left|\frac{\alpha_{1}+\left(\alpha_{2}-1\right)-\alpha_{1} \alpha_{2}}{\alpha_{1} \alpha_{2}}\right| & \nless 0, \\
\left|\frac{\alpha_{1}\left(1-\alpha_{2}\right)+\left(\alpha_{2}-1\right)}{\alpha_{1} \alpha_{2}}\right| & \nless 0, \\
\left|\frac{\left(1-\alpha_{2}\right)\left(\alpha_{1}-1\right)}{\alpha_{1} \alpha_{2}}\right| & \nless 0, \\
-\left(\frac{\left(1-\alpha_{2}\right)\left(\alpha_{1}-1\right)}{\alpha_{1} \alpha_{2}}\right) & \nless 0, \\
\left(\alpha_{2}-1\right)\left(\alpha_{1}-1\right) & \nless 0,
\end{aligned}
$$

since $\alpha_{1}>1$ and $\alpha_{2}>1$, we have a contradiction. Therefore, from Theorem. 2.2, the condition (i) is not satisfied and this is sufficient to conclude that the equilibrium point $E_{1}$ unstable. Thus, there is no need to test the conditions (ii) and (iv).

\section{Global Attracting of Fixed point}

Theorem 6.1. The equilibrium point $E_{0}$ is globally attracting if $\beta \neq 0$ is satisfied.

Proof. Let $f:(0, \infty) \times(0, \infty) \longrightarrow(0, \infty)$ and $g:(0, \infty) \times(0, \infty) \longrightarrow(0, \infty)$ are continuous functions. In addition, suppose that $f:\left[a_{1}, b_{1}\right] \times\left[a_{2}, b_{2}\right] \longrightarrow\left[a_{1}, b_{1}\right]$ and $g:\left[a_{1}, b_{1}\right] \times\left[a_{2}, b_{2}\right] \longrightarrow$ $\left[a_{2}, b_{2}\right]$, where $a_{1}, a_{2}, b_{1}$ and $b_{2}$ are positive real numbers. Such that

$$
f(\chi, \tau)=\frac{\alpha_{1} \chi \tau}{\beta+\tau} \quad \text { and } \quad g(\chi, \tau)=\frac{\alpha_{2} \chi \tau}{\beta+\chi} .
$$


Hence,

$$
\begin{aligned}
\frac{\partial f}{\partial \chi}=\frac{\alpha_{1} \tau}{(\beta+\tau)}, & \frac{\partial f}{\partial \tau}=\frac{\alpha_{1} \beta \chi}{(\beta+\tau)^{2}} \\
\frac{\partial g}{\partial \chi}=\frac{\alpha_{2} \beta \tau}{(\beta+\chi)^{2}}, & \frac{\partial g}{\partial \tau}=\frac{\alpha_{2} \chi}{(\beta+\chi)} .
\end{aligned}
$$

We observe that $f(\chi, \tau)$ and $g(\chi, \tau)$ are non decreasing in $\chi$ and $\tau$. Then Suppose that $m_{1}, m_{2}, M_{1}, M_{2}$ are positive real numbers such that $m_{1}=f\left(m_{1}, m_{2}\right), M_{1}=f\left(M_{1}, M_{2}\right), m_{2}=$ $g\left(m_{1}, m_{2}\right)$ and $M_{2}=g\left(M_{1}, M_{2}\right)$.

$$
m_{1}=\frac{\alpha_{1} m_{1} m_{2}}{\beta+m_{2}} \quad M_{1}=\frac{\alpha_{1} M_{1} M_{2}}{\beta+M_{2}},
$$

and

$$
m_{2}=\frac{\alpha_{2} m_{1} m_{2}}{\beta+m_{1}} \quad M_{2}=\frac{\alpha_{2} M_{1} M_{2}}{\beta+M_{1}} .
$$

From (17), we have

$$
\begin{aligned}
\left(1-\alpha_{1}\right) m_{1} m_{2}+\beta m_{1} & =0 \\
\left(1-\alpha_{1}\right) M_{1} M_{2}+\beta M_{1} & =0 .
\end{aligned}
$$

Multiply (19) by $M_{1} M_{2}$ and (20) by $m_{1} m_{2}$, we get

$$
\begin{aligned}
& \left(1-\alpha_{1}\right) m_{1} m_{2} M_{1} M_{2}+\beta m_{1} M_{1} M_{2}=0, \\
& \left(1-\alpha_{1}\right) M_{1} M_{2} m_{1} m_{2}+\beta M_{1} m_{1} m_{2}=0 .
\end{aligned}
$$

By subtracting (21) from (22), we obtain

$$
\beta\left(m_{1} M_{1} M_{2}-M_{1} m_{1} m_{2}\right)=0,
$$

since $\beta \neq 0$,

$$
m_{1} M_{1} M_{2}=M_{1} m_{1} m_{2} \quad \Rightarrow \quad M_{2}=m_{2} .
$$

Similarly, we get $M_{1}=m_{1}$. Then, from Theorem. 2.3, there exist only one equilibrium point and it is globally attractor.

\section{NUMERICAL EXAMPLES AND DISCUSSION}

In this section, we present some examples to see the effect of $\alpha_{1}, \alpha_{2}$ and $\beta$ in the behavior of the solution. We fix the initial values as $\chi_{-1}=3, \chi_{0}=4, \tau_{-1}=1$ and $\tau_{0}=2$.

Example. 1: We set $\alpha_{1}=2.5, \alpha_{2}=1.6$ and $\beta=2$. The result is given in Figure 1.

Example. 2: We have $\alpha_{1}=2.5, \alpha_{2}=1.6$ and $\beta=0.8$. The result is shown in Figure 2. 


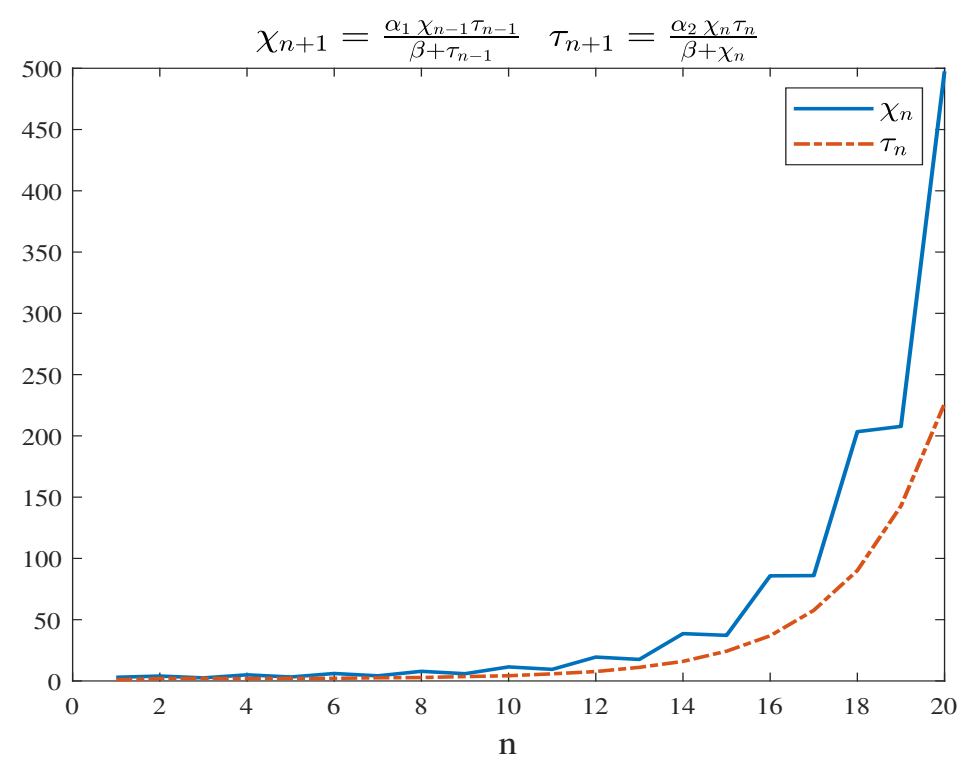

Figure 1. Plot the solution of system (1)

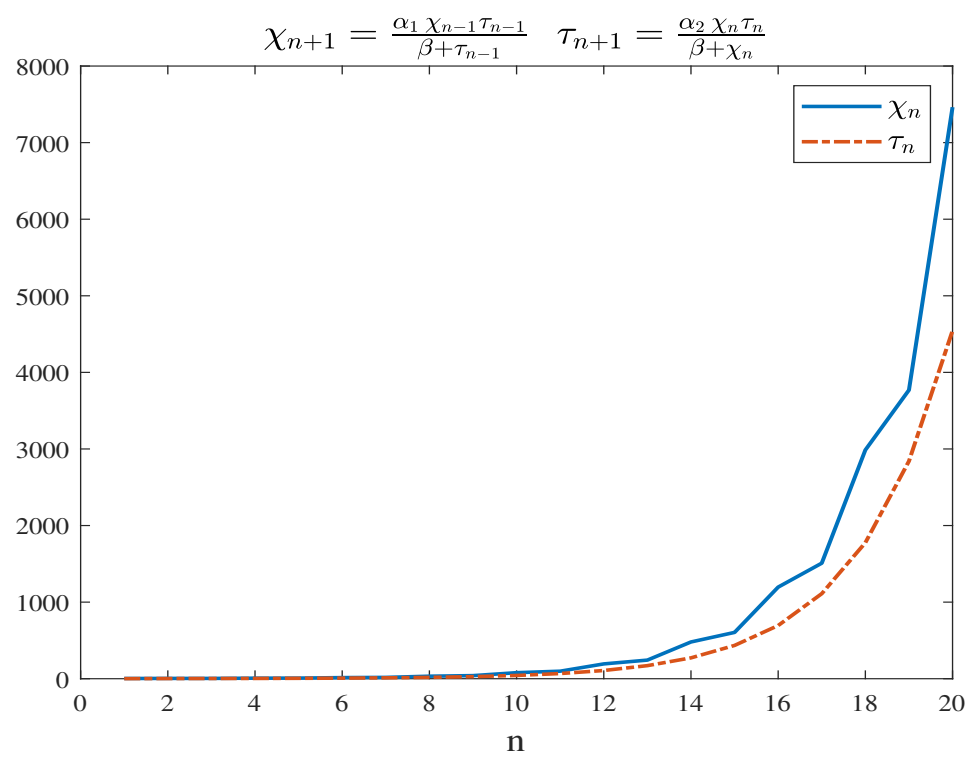

Figure 2. Plot the solution of system (1)

Example. 3: We set $\alpha_{1}=0.5, \alpha_{2}=0.6$ and $\beta=2$. The result is given in Figure 3 .

Example. 4: We have $\alpha_{1}=0.5, \alpha_{2}=0.6$ and $\beta=0.4$. The result is presented in Figure 4. 


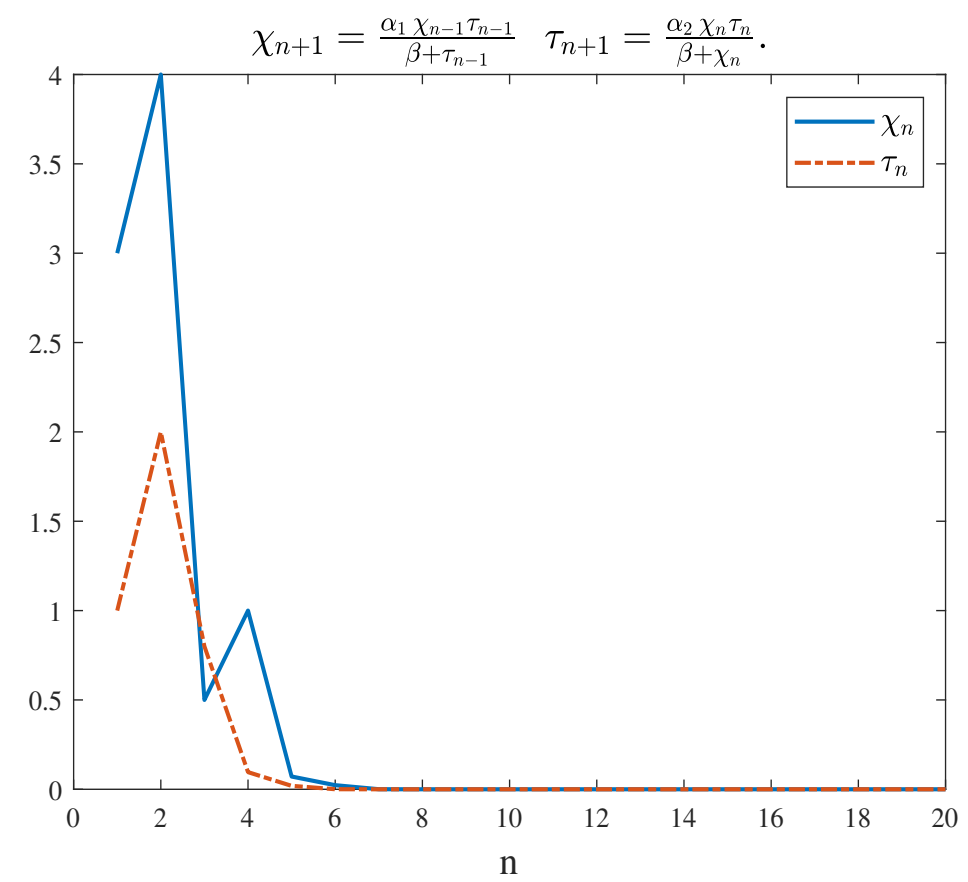

Figure 3. Plot the solution of system (1)

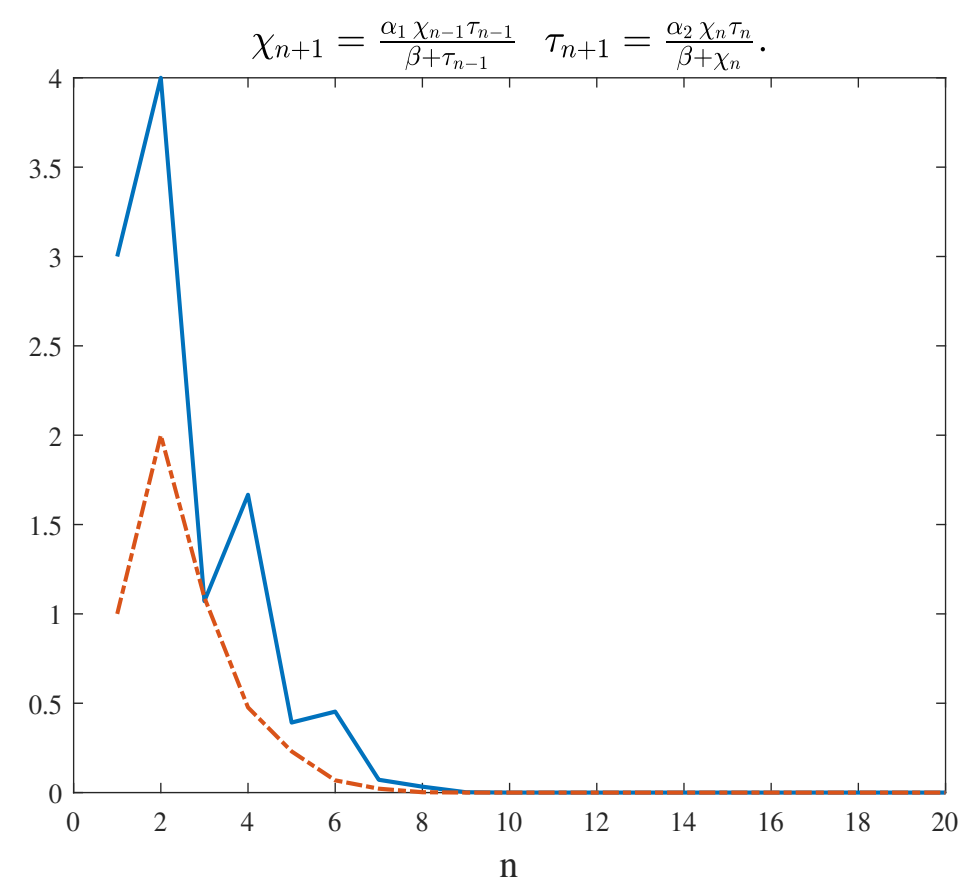

Figure 4. Plot the solution of system (1)

Example. 5: We fix $\alpha_{1}=1.5, \alpha_{2}=0.3$ and $\beta=2$. The result is given in Figure 5 .

Example. 6: We choose $\alpha_{1}=0.1, \alpha_{2}=1.6$ and $\beta=2$. The result is given in Figure 6. From Figure 1 and Figure 2, we see that when $\alpha_{1}$ and $\alpha_{2}$ are more than one, the solutions move to infinity while in Figure 3 and Figure 4 the solutions tend to the origin since $\alpha_{1}$ and $\alpha_{2}$ are less than one. It means the solutions are bounded and the equilibrium point $E_{0}$ is globally stable if and only if $\alpha_{1}$ and $\alpha_{2}$ are less than one. This result confirms with Theorem 3.1 and Theorem. 6.1. Moreover, we observe that the value of $\beta$ does not affect the state of stability 


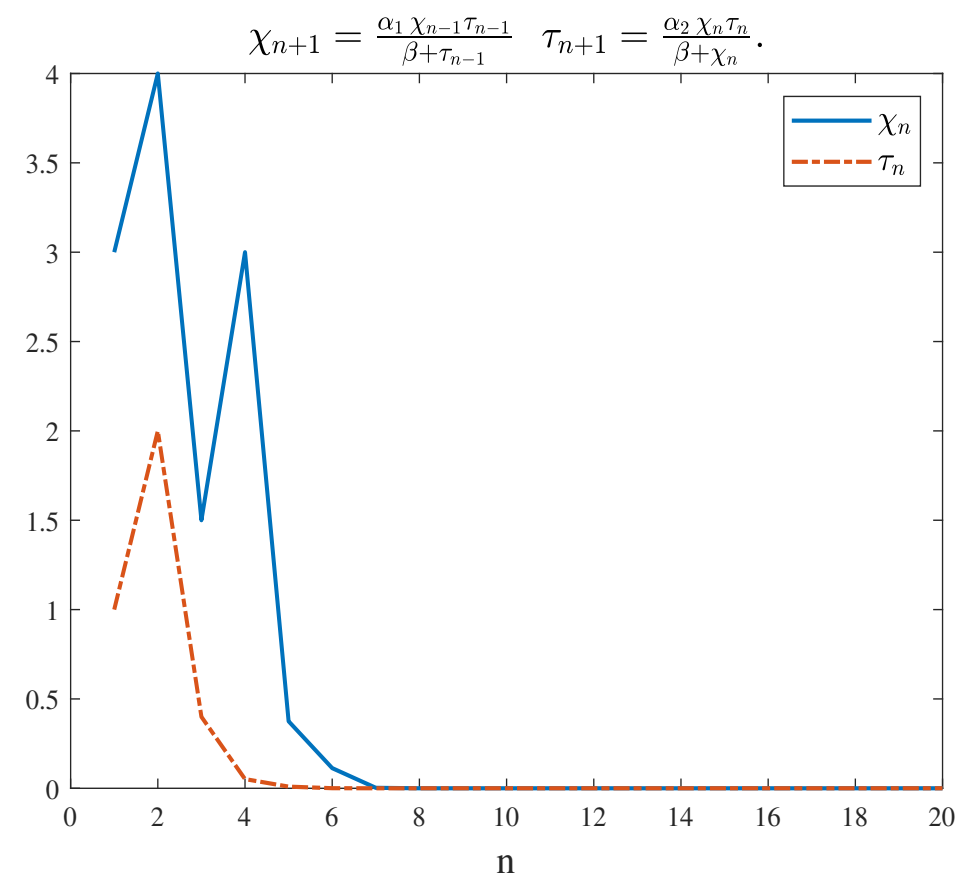

Figure 5. Plot the solution of system (1)

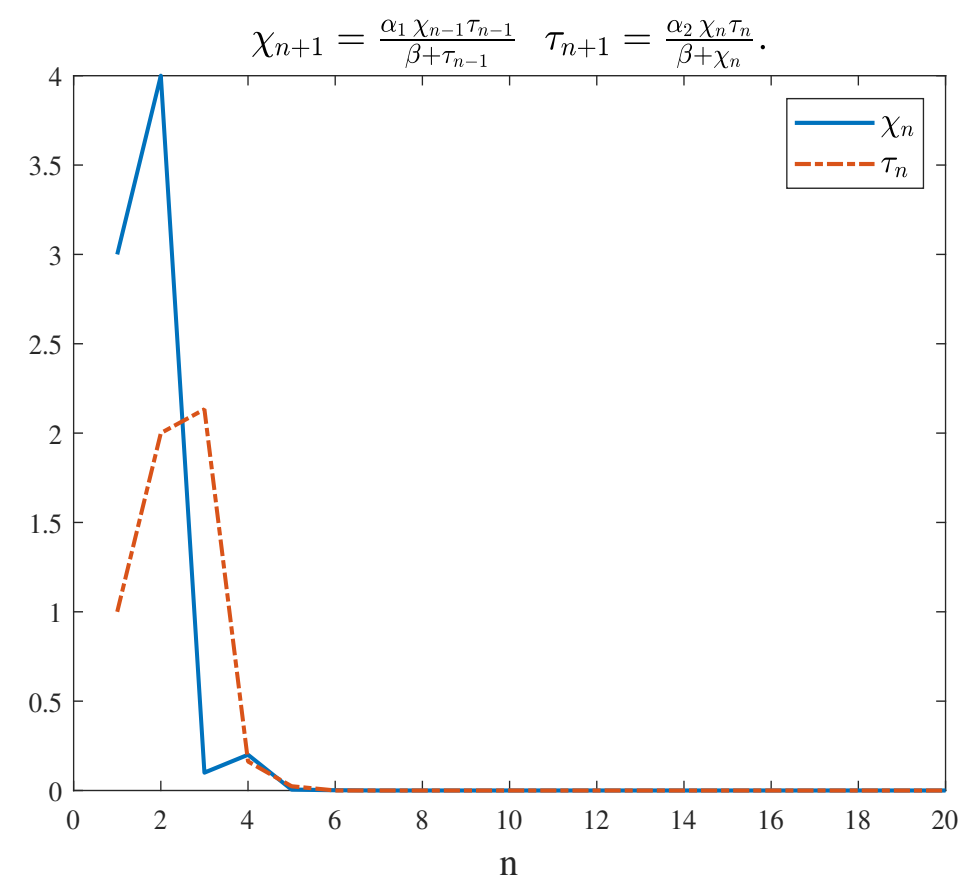

Figure 6. Plot the solution of system (1)

of the fixed point. Finally, from Figure 5 and Figure 6, we conclude that if $\alpha_{1}<1$ or $\alpha_{2}<1$, then the solutions are bounded and $E_{0}$ is globally stable.

\section{REFERENCES}

[1] M. Abd El-Moneam, T. Ibrahim and S. O. Alamoudy, Stability of a fractional difference equation of high order, J. Nonlinear Sci. Appl. 12 (2019) 65-74. https://doi.org/10.22436/jnsa.012.02.01.

[2] B. Al-Hdaibat, S. Al-Ashhab and R. Sabra, Explicit solutions and bifurcations for a system of rational difference equations, Mathematics. 7 (2019) 69. https://doi.org/10.3390/math7010069. 
[3] M. M. El-Dessoky and E. M. Elabbasy and A. Asiri, Dynamics and solutions of a fifth-order nonlinear difference equation, Discrete Dyn. Nat. Soc. 2018 (2018) 9129354. https://doi.org/10.1155/2018/9129354.

[4] S. Elaydi, An Introduction to Difference Equation, Springer-Verlag New York, NY, USA, Third edition. 2005. https://doi.org/10.1007/0-387-27602-5.

[5] E. M. Elsayed, Solutions of rational difference systems of order two, Math. Comput. Model. 55 (2012) 378-384. https://doi.org/10.1016/j.mcm.2011.08.012.

[6] E. M. Elsayed and A. M. Ahmed, Dynamics of a three-dimensional systems of rational difference equations, Math. Methods Appl. Sci. 39 (2016) 1026-1038.https://doi.org/10.1002/mma.3540.

[7] E. M. Elsayed and F. Alzahrani, Periodicity and solutions of some rational difference equations systems, J. Appl. Anal. Comput. 9 (2019) 2358-2380,https://doi.org/10.11948/20190100.

[8] E. A. Grove and G. Ladas, Periodicities in Nonlinear Difference Equations, Chapman and Hall/CRC. First edition. 2004.

[9] M. Gümüs and R. Abo-Zied, Global behavior of a rational second order difference equation, J. Appl. Math. Comput. 62 (2020) 119-133. https://doi.org/10.1007/s12190-019-01276-9.

[10] N. Haddad, N. Touafek, J.F.T. Rabago, Well-defined solutions of a system of difference equations, J. Appl. Math. Comput. 56 (2018) 439-458. https://doi.org/10.1007/s12190-017-1081-8.

[11] M. Kara and Y. Yazlik, Solvability of a system of nonlinear difference equations of higher order, Turk. J. Math. 43 (2019) 1533-1565. https://doi.org/10.3906/mat-1902-24.

[12] M. Kara and Y. Yazlik and D. Tollu, Solvability of a system of higher order nonlinear difference equations, Hacettepe J. Math. Stat. 49 (2020) 1566-1593.https://doi.org/10.15672/hujms . 474649.

[13] A. Khaliq and M. Shoaib, Dynamics of three-dimensional system of second order rational difference equation, Electron. J. Math. Anal. Appl. 9 (2021) 308-319.

[14] M. S. Khan, Q. Din, M. Habib and M. A. Khan, Stability analysis of a system of second order rational difference equations, Comput. Ecol. Software. 10 (2020) 44-58.

[15] A. Kurbanli, C. Cinar and İ. Yalcinkaya, On the behavior of positive solutions of the system of rational difference equations $x_{n+1}=x_{n} /\left(y_{n} x_{n}+1\right) y_{n+1}=y_{n} /\left(x_{n} y_{n}+1\right)$, Math. comput. model. 53 (2011) 12611267. https://doi.org/10.1016/j.mcm.2010.12.009.

[16] O. Moaaz, G. E. Chatzarakis, D. Chalishajar and O. Bazighifan, Dynamics of general class of difference equations and population model with two age classes, Mathematics. 8 (2020) 516. https://doi.org/10. $3390 /$ math8040516. 